\title{
Rheumatic diseases induced by drugs and environmental factors: the state-of-the-art - part two
}

\author{
Karolina Niklas ${ }^{1}$, Arkadiusz A. Niklas ${ }^{2}$, Dominik Majewski ${ }^{1}$, Mariusz J. Puszczewicz ${ }^{1}$ \\ ${ }^{1}$ Department of Rheumatology and Internal Diseases, Poznan University of Medical Science, Poland \\ ${ }^{2}$ Department of Hypertension, Angiology and Internal Medicine, Poznan University of Medical Science, Poland
}

\begin{abstract}
The majority of rheumatic diseases belong to the group of autoimmune diseases and are associated with autoantibody production. Their etiology is not fully understood. Certain medications and environmental factors may have an influence on the occurrence of rheumatic diseases. Establishing a cause-effect relationship between a certain factor and disease induction is not always simple. It is important to administer the drug continuously or monitor exposure to a given factor in the period preceding the onset of symptoms. The lack of early diagnosed autoimmune disease, or finally the lack of symptoms within a few weeks/months after discontinuation of the drug/cessation of exposure, is also important.

The most frequently mentioned rheumatic diseases caused by drugs and environmental factors include systemic lupus erythematosus (SLE), scleroderma, systemic vasculitis, polymyositis, dermatomyositis, and Sjögren's syndrome.

The objective of this study is to summarize current knowledge on rheumatic diseases induced by drugs and environmental factors.
\end{abstract}

Key words: rheumatic diseases, drugs, environmental factors.

\section{Introduction}

The objective of this study is to summarize current knowledge on rheumatic diseases induced by drugs and environmental factors. We performed a literature review from 2010 to 2015 using PubMed. In some reasonable cases we also included previous publications (especially in the situation of historical value). Publications containing the key words "drug induced \& name of disease" and "environmental factors \& name of disease" were selected, and as the name of the disease in the second part of our paper we used: vasculitis, polymyositis, dermatomyositis, rheumatoid arthritis, Sjögren's syndrome.

\section{Drug-induced vasculitis}

Vasculitis is a heterogeneous group of disease entities, whose common feature is vascular wall damage through inflammation caused by autoimmune processes.
The severity of the disease depends on whether only skin and subcutaneous tissue vessels are occupied or whether organ alterations occur.

Vasculitis can also be induced by numerous drugs including non-steroidal anti-inflammatory drugs (NSAIDs), antibiotics (among them minocycline is the most commonly described), antihypertensive drugs, propylthiouracil, and anticonvulsants. An association between leukotrienes - medicines used to treat asthma - and the occurrence of Churg-Strauss syndrome is known. Some authors, however, claim that introduction of these drugs can reduce the dosage of corticosteroids in patients with asthma, and consequently, disease previously masked by immunosuppressants is revealed. However, there are also known individual cases in which the glucocorticoid dosage remained unchanged, even though patients presented with ChurgStrauss syndrome after implementation of leukotriene [1]. Among newer medications that can cause vasculitis, spe- 
cial attention is paid to TNF inhibitors and new small molecules, i.e. kinase inhibitors: gefitinib used in oncology and masitinib, analyzed in terms of the treatment effectiveness in rheumatoid arthritis [2, 3].

Usually, the symptoms of vasculitis associated with drugs appear 7-10 days after drug inclusion, although sometimes they become apparent after several months of pharmaceutical administration. Symptoms resemble primary vasculitis. Skin lesions generally include lower limbs and buttocks forming palpable purpura, even though one can observe urticaria-like alterations. Necrotic lesions can also be observed. Symptoms such as weakness, fatigue, and fever are frequent. In addition, one may observe muscle and joint pain, and sometimes internal organ failure, most commonly lungs and kidneys (in extreme cases, even bleeding into the alveolar spaces has been reported). Cases of localized inflammation in the coronary arteries with subsequent heart failure have also been known to occur $[4,5]$.

In laboratory tests, neutrophilia is usually observed, sometimes eosinophilia. Anti-neutrophil cytoplasmic antibodies (ANCA) are the most commonly reported autoantibodies. While in the primary vasculitis these are predominantly directed against single neutrophil cytoplasmic antigens, in drug-induced vasculitis antibodies against many antigens are often present. Among these, myeloperoxidase (MPO), human leukocyte elastase (HLE), cathepsin G, lactoferrin and azurocidin are mentioned. It has also been observed that in drug-induced inflammation MPO-ANCA titers are significantly higher than in primary inflammations.

Furthermore, a strong association between exposure to drugs and the presence of HLE-ANCA and ANCA directed against lactoferrin [6] has been reported. Other antibodies that may occur in drug-induced vasculitis are anti-histone antibodies and antiphospholipid antibodies. Their presence and the concurrent presence of HLE-ANCA or ANCA directed against several antigens should increase diagnostic sensitivity towards vasculitis induced by drugs [5].

Treatment is based on discontinuation of the drugs causing the diseases. If alterations are restricted only to the skin, most commonly they disappear completely, and the prognosis is good. In the case of organ changes, glucocorticoids are included the most frequently. Heart or kidney failure may be associated with $30 \%$ mortality [4].

\section{Vasculitis versus environmental factors}

Vasculitis can also be caused by environmental factors. Infectious agents are primarily mentioned [7]. Viral infections rank first. It is known that polyarteritis nodosa is accompanied in 10-15\% of cases by hepatitis B virus $(\mathrm{HBV})$. Also, the presence of hepatitis C virus ( $\mathrm{HCV}$ ) has been observed in patients with mixed cryoglobulinemia with concurrent vasculitis [4]. D’Alessandro et al. [8] described a case of a 3-year-old girl who developed full-blown Henoch-Schönlein purpura caused by cytomegalovirus (CMV). Another virus which may be associated with the occurrence of vasculitis is B19 parvovirus, although no clear evidence has been reported [9]. Also, bacterial antigens are taken into account in the etiology of vasculitis. These include Streptococcus spp., Staphylococcus aureus and Gram-negative bacteria antigens $[4,10]$. Staphylococcus aureus is particularly associated with the occurrence of granulomatosis with vasculitis (formerly known as Wegener's granulomatosis) [9].

Another important environmental factor which may cause vasculitis is, similar to scleroderma, silica dust. Inhaled dust can cause not only lung macrophage activation with subsequent stimulation of fibroblasts, but also $T$ cell stimulation resulting in neutrophil accumulation, which is the source of MPO, against which antibodies reported in vasculitis are observed [9]. An association between exposure to silica dust and vasculitis was also confirmed in a meta-analysis by Gómez-Puerta et al. [11].

\section{Drug-induced polymyositis and dermatomyositis}

Polymyositis (PM) and dermatomyositis (DM) are systemic connective tissue diseases characterized primarily by muscle weakness, muscle pain and, in DM, additionally by skin lesions. In both forms, common symptoms such as fatigue, raised temperature, and weight loss are frequent. Other symptoms include arthralgia, swollen joints and internal organ failure, including the lungs, heart and gastrointestinal tract. In laboratory tests, elevated CRP concentrations and markers of muscle damage are observed: increased activity of phosphocreatine kinase (CPK), aspartate aminotransferase (AST), alanine aminotransferase (ALT), aldolase, and lactate dehydrogenase (LDH). Among characteristic antibodies present in the serum of patients, one can find anti-tRNA synthetase antibodies, and among them anti-Jo-1. Among other antibodies, anti-nuclear helicase antibodies (anti-Mi-2) against TIF1- $\gamma$ protein (anti-p155/140), or against molecules recognizing the signal (anti-SRP), are important.

Both PM and DM can be induced by drugs. The most frequently mentioned drugs are TNF inhibitors including etanercept and adalimumab. These drugs cause fullblown disease with the occurrence of anti-Jo-1 antibodies, but there is also a case of a patient with clinical symptoms without the presence of antibodies in serum [2, 12]. Liu et al. [13] presented a case of a 46-year-old patient who reported DM symptoms after etanercept administration. Interestingly, alterations subsided after discontinuation of the treatment and returned again after administration 
of adalimumab. Only total withdrawal of anti-TNF therapy resulted in sustained improvement in DM [13].

Another group of drugs associated with the induction of PM and DM is the lipid-lowering drug group, mainly statins, but a case of PM caused by gemfibrozil has also been reported. Statins may be a cause of the whole spectrum of muscle damage from focal lesions to full-blown DM $[2,14,15]$. Other drugs that cause myositis are hydroxyurea, which in 70 publications investigated by Seidler and Gottlieb [16] accounted for 51\% of DM cases, but also interferons, gonadoliberin, D-penicillamine, the tyrosine kinase inhibitor sorafenib [2]; a new drug used to treat hepatitis B - clevudine [17]; ipilimumab - a human monoclonal antibody used in the treatment of metastatic melanoma [18]; and zoledronic acid, prescribed for the treatment of osteoporosis [19].

\section{Polymyositis and dermatomyositis versus environmental factors}

Among the environmental factors that may trigger PM/DM, infectious agents are mentioned in the first place, especially Coxsackie B virus [20]. Bacterial and parasitic infections such as trichinosis or toxoplasmosis can also lead to muscle damage [21].

An interesting paper about the impact of environmental factors on the occurrence of PM was presented by Takenaka et al. [22]. They described a case of a 73-year-old farmer, who was diagnosed with polymyositis and for whom effective therapy with glucocorticoids was implemented. However, the authors were intrigued that in a short time similar symptoms occurred in his 44-year-old son after moving to his parents' house and replacing his father on his farm during the period of his father's hospitalization. Unfortunately, the agent responsible for the occurrence of the disease was not identified; however, one thesis put forward was that environmental factors and genetic susceptibility have great importance in the induction of PM [22].

\section{Other rheumatic diseases and their association with drugs and environmental factors}

In the literature, there are also reports on other rheumatic diseases and their associations with drugs or environmental factors.

An example is Sjögren's syndrome, for which the occurrence has been associated with the use of hydralazine [23] and phenobarbital [2]. Among environmental factors, mainly viral infections, in particular, Epstein-Barr virus (EBV) and CMV [24], as well as exposure to acrylamide, have an influence on the induction of Sjögren's syndrome [25].
Some authors have suggested an association between rheumatoid arthritis (RA) and exposure to silica dust [11]. A well-known and one of the most important environmental risk factors for development and severity of RA is smoking [26]. Also air pollution is considered as being responsible for the occurrence of rheumatoid arthritis [27]. Another important environmental factor in pathogenesis of RA is periodontitis. It has also been associated with increased prevalence of many other diseases, e.g. cardiovascular diseases, diabetes, and inflammatory bowel disease. Periodontitis, which is a chronic reservoir of microparticles, may cause the passage of inflammatory cytokines or bacteria in the bloodstream, thus playing a role in initiation of systemic diseases [28].

Goldstein et al. [29] described two cases of polymyalgia rheumatica with concurrent giant cell arteritis in patients treated with ipilimumab due to malignant melanoma.

Environmental factors may also have an impact on the occurrence of undifferentiated connective tissue diseases (UCTD). UCTD occur when certain symptoms of connective tissue diseases appear, but no diagnostic criteria are met. Environmental factors that may induce UCTD include occupational exposure to solvents, perfumes, cosmetics, paints or working in a laboratory [30].

To summarize, we present the main factors inducing selected rheumatic diseases in Table I.

\section{Vaccinations and occurrence of rheumatic disease}

In the face of wide-ranging discussion on the safety of vaccination, the question as to whether they may be associated with the occurrence of rheumatic diseases arises.

In the literature, there is only a single case report on vaccination-induced RA against tetanus, influenza or poliomyelitis [30].

In the case of lupus, vaccines against influenza, hepatitis B or human papillomavirus (HPV) are mentioned among factors potentially responsible for its occurrence $[30,31]$. Simultaneously, the authors emphasize that vaccinations against HPV are usually conducted in adolescence, which is also characteristic for the occurrence of certain autoimmune diseases, including SLE, and it is not always possible to unambiguously determine whether this vaccination induced the disease.

A case of antiphospholipid syndrome, which occurred a few months after vaccination against diphtheria and tetanus, has also been described [32].

Slightly more vaccines are associated with the occurrence of vasculitis. Vaccination against influenza, tuberculosis, hepatitis B, and HPV [2, 30, 33, 34] are mentioned among these. 
Table I. The main factors which can induce selected rheumatic diseases

\begin{tabular}{|c|c|c|}
\hline & Drugs & Environmental factors \\
\hline $\begin{array}{l}\text { Systemic lupus } \\
\text { erythematosus }\end{array}$ & $\begin{array}{l}\text { procainamide } \\
\text { hydralazine } \\
\text { quinidine } \\
\text { estrogen } \\
\text { TNF inhibitors }\end{array}$ & $\begin{array}{c}\text { ultraviolet radiation } \\
\text { aromatic amines } \\
\text { silica dust } \\
\text { Epstein-Barr virus } \\
\text { human endogenous retroviruses }\end{array}$ \\
\hline Systemic sclerosis & $\begin{array}{l}\text { bleomycin } \\
\text { paclitaxel } \\
\text { pentazocine } \\
\text { cocaine }\end{array}$ & $\begin{array}{l}\text { silica dust } \\
\text { vinyl chloride } \\
\text { organic solvents } \\
\text { epoxy resins }\end{array}$ \\
\hline Vasculitis & $\begin{array}{c}\text { non-steroidal anti-inflammatory drugs } \\
\text { antibiotics } \\
\text { leukotrienes } \\
\text { TNF inhibitors }\end{array}$ & $\begin{array}{l}\text { hepatitis B virus } \\
\text { hepatitis C virus } \\
\text { cytomegalovirus } \\
\text { bacterial antigens } \\
\text { silica dust }\end{array}$ \\
\hline $\begin{array}{c}\text { Polymyositis } \\
\text { and dermatomyositis }\end{array}$ & $\begin{array}{l}\text { TNF inhibitors } \\
\text { statins } \\
\text { hydroxyurea }\end{array}$ & $\begin{array}{l}\text { Coxsackie B virus } \\
\text { bacterial infections } \\
\text { parasitic infections }\end{array}$ \\
\hline Rheumatoid arthritis & & $\begin{array}{l}\text { smoking } \\
\text { periodontitis } \\
\text { silica dust }\end{array}$ \\
\hline
\end{tabular}

Many vaccines have also been associated with the occurrence of PM/DM, including those against influenza, hepatitis B, varicella, mumps, rubella, poliomyelitis, tuberculosis, tetanus, diphtheria and pertussis [35] However, the authors highlight the fact that these are not frequent cases.

Soriano et al. [36] also reported an association between the application of vaccination and Sjögren's syndrome.

Post-vaccination phenomena may also be associated with exposure to an adjuvant. Similar symptoms are observed in siliconosis, Gulf war syndrome (GWS), and macrophagic myofasciitis syndrome (MMF). In 2011 Shoenfeld and Agmon-Levin [37] proposed to name these conditions autoimmune (auto-inflammatory) syndrome induced by adjuvants (ASIA).

It is obvious that, similar to natural infectious agents, vaccinations may cause symptoms of certain diseases, especially immune-related ones. However, it should be remembered that all the above-described cases are only incidental against the background of the number of vaccinations currently carried out. In many situations, the authors do not explicitly indicate the association between vaccination and the onset of the disease, and their deliberations are speculative. Therefore, it should be highlighted that such reports are not intended to negate the value of vaccinations, but only to note that some side effects may be present after their application, especially in genetically predisposed individuals.

\section{Summary}

Rheumatic diseases may be induced by drugs and other factors, including environmental factors. We must remember that the key is to eliminate the causative factor, in particular in the case of an abnormal laboratory or clinical picture, in patients who claim to administer drugs previously confirmed to be associated with the occurrence of autoimmune diseases, or in the case of exposure to harmful environmental factors, as well as in patients who do not respond to conventional therapy.

In 2010, the National Institute of Environmental Health Sciences (NIEHS) organized an expert panel workshop concerning the role of environmental factors in autoimmune diseases. It took into consideration not only rheumatic diseases but also type 1 diabetes, celiac disease, multiple sclerosis, Crohn's disease, Graves' disease and many others. One of the conclusions was that interdisciplinary research is still needed to clarify the role of environmental factors in autoimmune diseases [38].

The authors declare no conflict of interest.

\section{References}

1. Uyar M, Elbek O, Bakır K, et al. Churg-Strauss syndrome related to montelukast. Tuberk Toraks 2012; 60: 56-58.

2. Bukhari M. Drug-induced rheumatic diseases: a review of published case reports from the last two years. Curr Opin Rheumatol 2012; 24: 182-186. 
3. Grau RG. Drug-induced vasculitis: new insights and a chang ing lineup of suspects. Curr Rheumatol Rep 2015; 17: 71.

4. D'Cruz D. Autoimmune diseases associated with drugs, chemicals and environmental factors. Toxicol Lett 2000; 112-113: 421-432.

5. Wiik A. Drug-induced vasculitis. Curr Opin Rheumatol 2008, 20: 35-39.

6. Choi HK, Merkel PA, Walker AM, et al. Drug-associated antineutrophil cytoplasmic antibody-positive vasculitis: prevalence among patients with high titers of antimyeloperoxidase antibodies. Arthritis Rheum 2000; 43: 405-413.

7. Muñoz-Grajales C, Pineda JC. Pathophysiological relationship between infections and systemic vasculitis. Autoimmune Dis 2015; 2015: 286783.

8. D’Alessandro M, Buoncompagni A, Minoia F, et al. Cytomegalovirus-related necrotising vasculitis mimicking Henoch-Schönlein syndrome. Clin Exp Rheumatol 2014; 32 (3 Suppl 82) S73-75.

9. Chen M, Kallenberg CG. The environment, geoepidemiology and ANCA-associated vasculitides. Autoimmun Rev 2010; 9 A293-298.

10. Kallenberg CG. Pathogenesis of ANCA-associated vasculitides Ann Rheum Dis 2011; 70 Suppl 1: i59-63.

11. Gómez-Puerta JA, Gedmintas L, Costenbader KH. The association between silica exposure and development of ANCA-associated vasculitis: systematic review and meta-analysis. Autoimmun Rev 2013; 12: 1129-1135.

12. Brunasso AM, Scocco GL, Massone C. Dermatomyositis during adalimumab therapy for rheumatoid arthritis. J Rheumato 2010; 37: 1549-1550.

13. Liu SW, Velez NF, Lam C, et al. Dermatomyositis induced by anti-tumor necrosis factor in a patient with juvenile idiopathic arthritis. JAMA Dermatol 2013; 149: 1204-1208.

14. Hahn M, Sriharan K, McFarland MS. Gemfibrozil-induced myositis in a patient with normal renal function. Ann Pharmacother 2010; 44: 211-214.

15. Niklas K, Niklas A, Puszczewicz M, et al. Polymyositis induced by atorvastatin. Kardiol Pol 2015; 73: 1336.

16. Seidler AM, Gottlieb AB. Dermatomyositis induced by drug therapy: a review of case reports. J Am Acad Dermatol 2008, 59: 872-880

17. Yang CY, Park SA, Kim HS, et al. Polymyositis in patients taking antiviral clevudine therapy: a report of two cases. NeuroRehabilitation 2010; 26: 159-162.

18. Sheik Ali S, Goddard AL, Luke JJ, et al. Drug-associated dermatomyositis following ipilimumab therapy: a novel immune-mediated adverse event associated with cytotoxic T-lymphocyte antigen 4 blockade. JAMA Dermatol 2015; 151: 195-199.

19. Tong PL, Yu LL, Chan JJ. Drug-induced dermatomyositis after zoledronic acid. Australas J Dermatol 2012; 53: e73-75.

20. Pignone A, Fiori G, Del Rosso A, et al. The pathogenesis of inflammatory muscle diseases: on the cutting edge among the environment, the genetic background, the immune response and the dysregulation of apoptosis. Autoimmun Rev 2002; 1 : 226-232.

21. Majewski D, Puszczewicz M, Kołczewska A. Differential diagnosis of polymyositis. Case report. Ann Acad Med Stetin 2010; 56 Suppl 1: 66-69.
22. Takenaka K, Miyabe Y, Kasai S, et al. Polymyositis in a father and his son; a case report. Nihon Rinsho Meneki Gakkai Kaishi 2012; 35: 144-149.

23. Darwaza A, Lamey PJ, Connell JM. Hydrallazine-induced Sjögren's syndrome. Int J Oral Maxillofac Surg 1988; 17: 92-93.

24. Kivity S, Arango MT, Ehrenfeld M, et al. Infection and autoimmunity in Sjogren's syndrome: A clinical study and comprehensive review. J Autoimmun 2014; 51: 17-22.

25. Rothschild B. Acrylamine-induced autoimmune phenomena. Clin Rheumatol 2010; 29: 999-1005.

26. Chang K, Yang SM, Kim SH, et al. Smoking and rheumatoid arthritis. Int J Mol Sci 2014; 15: 22279-22295.

27. Essouma M, Noubiap JJ. Is air pollution a risk factor for rheumatoid arthritis? J Inflamm (Lond) 2015; 12: 48.

28. Badran Z, Struillou X, Verner C, et al. Periodontitis as a risk factor for systemic disease: are microparticles the missing link? Med Hypotheses 2015; 84: 555-556.

29. Goldstein BL, Gedmintas L, Todd DJ. Drug-associated polymyalgia rheumatica/giant cell arteritis occurring in two patients after treatment with ipilimumab, an antagonist of CTLA-4. Arthritis Rheumatol 2014; 66: 768-769.

30. Zandman-Goddard G, Solomon M, Rosman Z, et al. Environment and lupus-related diseases. Lupus 2012; 21: 241-250.

31. Pellegrino P, Carnovale C, Pozzi M, et al. On the relationship between human papilloma virus vaccine and autoimmune diseases. Autoimmun Rev 2014; 13: 736-741.

32. Meyer A, Rotman-Pikielny P, Natour A, et al. Antiphospholipid syndrome following a diphtheria-tetanus vaccination: coincidence vs. causality. Isr Med Assoc J 2010; 12: 638-639.

33. Ghattaura A, Eley KA, Molenaar E, et al. A case of extensive ulcerating vasculitis following a BCG vaccination. J Plast Reconstr Aesthet Surg 2009; 62: e286-289.

34. Le Hello C, Cohen P, Bousser MG, et al. Suspected hepatitis B vaccination related vasculitis. J Rheumatol 1999; 26: 191-194.

35. Stübgen JP. A review on the association between inflammatory myopathies and vaccination. Autoimmun Rev 2014; 13 : 31-39.

36. Soriano A, Afeltra A, Shoenfeld Y. Immunization with vaccines and Sjögren's syndrome. Expert Rev Clin Immunol 2014; 10: 429-435.

37. Shoenfeld Y, Agmon-Levin N. 'ASIA'-autoimmune/inflammatory syndrome induced by adjuvants. J Autoimmun 2011; 36: 4-8.

38. Parks CG, Miller FW, Pollard KM, et al. Expert panel workshop consensus statement on the role of the environment in the development of autoimmune disease. Int J Mol Sci 2014; 15 : 14269-14297. 